\title{
Pulsed Dipolar Spectroscopy Reveals that Tyrosyl Radicals are generated in Both Monomers of the Cyclooxygenase-2 Dimer
}

\author{
Benjamin J. Orlando ${ }^{1, \ddagger}$, Peter P. Borbat ${ }^{2,3, \ddagger}$, Elka R. Georgieva ${ }^{2,3, \ddagger}$, Jack H. Freed $^{2,3}$, and \\ Michael G. Malkowski ${ }^{1,4,{ }^{*}}$ \\ ${ }^{1}$ Department of Structural Biology, The State University of New York at Buffalo, Buffalo, NY \\ 14203, USA \\ ${ }^{2}$ National Biomedical Center for Advanced Electron Spin Resonance Technology, Cornell \\ University, Ithaca, NY, USA \\ ${ }^{3}$ Department of Chemistry and Chemical Biology, Cornell University, Ithaca, NY, USA \\ ${ }^{4}$ Hauptman-Woodward Medical Research Institute, Buffalo, NY 14203, USA
}

\begin{abstract}
Cyclooxygenases (COX) are heme containing sequence homodimers that utilize tyrosyl radicalbased catalysis to oxygenate substrates. Tyrosyl radicals are formed from a single turnover of substrate in the peroxidase active site generating an oxy-ferryl porphyrin cation radical intermediate that subsequently gives rise to a Tyr-385 radical in the cyclooxygenase active site and a Tyr-504 radical nearby. We have utilized double quantum coherence (DQC) spectroscopy to determine the distance distributions between Tyr-385 and Tyr-504 radicals in COX-2. The distances obtained with DQC confirm that Tyr-385 and Tyr-504 radicals were generated in each monomer and accurately match the distances measured in COX-2 crystal structures.
\end{abstract}

The cyclooxygenases (COX-1 and COX-2) are the primary targets of non-steroidal antiinflammatory drugs (NSAIDs) and COX-2 selective inhibitors (coxibs). COX enzymes are heme containing sequence homodimers. Each monomer contains two active sites: a cyclooxygenase active site that catalyzes the bis-dioxygenation of arachidonic acid (AA) to form the intermediate prostaglandin $\mathrm{G}_{2}\left(\mathrm{PGG}_{2}\right)$, and a peroxidase active site that reduces the 15-hydroperoxide group of $\mathrm{PGG}_{2}$ to form $\mathrm{PGH}_{2}$. $\mathrm{COX}$ requires a preliminary catalytic turnover at the peroxidase active site to generate an oxy-ferryl porphyrin cation radical (Fig. S1). The radical is subsequently transferred to Tyr-385 located in the cyclooxygenase active site, which abstracts the 13-proS hydrogen from AA to initiate cyclooxygenase catalysis (Fig S1) ${ }^{1}$. $\mathrm{PGH}_{2}$ is metabolized by downstream tissue specific synthases to form potent lipid

\footnotetext{
Corresponding Author, Michael G. Malkowski, Department of Structural Biology, State University of New York at Buffalo, 700 Ellicott Street, Buffalo, NY 14203, USA.mgm22@buffalo.edu.

*These authors contributed equally.

No competing financial interests have been declared.

Supporting Information

Supplementary methods and figures related to protein production and DQC analyses is available free of charge online at http:// pubs.acs.org.
} 
signaling molecules involved in regulating physiological homeostasis. These molecules also play intricate roles in pathological states such as inflammation and cancer ${ }^{1,2}$.

ESR spectroscopic studies have shown that a radical is also formed on Tyr-504 during cyclooxygenase catalysis ${ }^{3,4}$. Reaction of $\mathrm{Y} 385 \mathrm{~F} \mathrm{COX}-2$ with a peroxide substrate generates a radical that is localized solely on Tyr-504 ${ }^{4}$. Tyr-504 is located near the proximal heme ligand just outside of the cyclooxygenase active site (Fig S2) and is not directly involved in catalysis, as Y504F COX-2 retains wild-type levels of activity ${ }^{4}$. While the role of the Tyr-504 radical is not clear, it has been proposed by Rogge and colleagues that an equilibrium exists between the radicals on Tyr-385 and Tyr-504 (Fig S2), with Tyr-504 serving as a "radical reservoir" that replenishes the catalytic Tyr-385 radical after depletion by reductant ${ }^{4}$. Importantly, previous continuous wave ESR studies have not provided information on the spatial location of tyrosyl radicals with respect to one another in the COX dimer. Mutational studies using a heterodimer of COX-2 in which one monomer lacked peroxidase activity and the partner monomer lacked cyclooxygenase activity resulted in a cyclooxygenase deficient enzyme, demonstrating that electron transfer across the dimer interface does not occur ${ }^{5}$. Thus, for the radical reservoir hypothesis to hold true, Tyr-385 and Tyr-504 radicals must be generated within the same monomer, which has yet to be demonstrated experimentally.

A new paradigm has emerged with respect to COX catalysis and regulation. In this model, COX functions as a conformational heterodimer with only one monomer active at a given time $^{6}$. Dietary nonsubstrate fatty acids and certain NSAIDs bind to one monomer, the "allosteric" monomer $\left(\mathrm{E}_{\text {allo }}\right)$, to modulate substrate oxygenation in the partner "catalytic" monomer $\left(\mathrm{E}_{\mathrm{cat}}\right)^{6,7}$. The mechanism governing inter-monomer communication is unknown and it remains unclear if tyrosyl radicals play a role in allosteric regulation. A potential scenario in which a catalytic Tyr-385 radical is generated in only one monomer has not been ruled out (Fig. S2) ${ }^{6,8}$. This scenario is particularly intriguing, as it would provide an explanation for the half-of-sites reactivity observed with COX-2. A complete understanding of COX half-of-sites reactivity and allostery is necessary, as current investigations are trending towards the design of next generation NSAIDs that inhibit COX in a substrateselective and allosteric manner ${ }^{9}$.

Pulsed ESR techniques such as double electron-electron resonance (DEER) and double quantum coherence (DQC) are powerful methods for extracting distance information between paramagnetic species in proteins ${ }^{10}$. While these techniques are often utilized in combination with nitroxide spin labels that are introduced into proteins via site-directed spin labeling, their utility has been demonstrated with additional paramagnetic cofactors. 4-pulse DEER has previously been utilized to determine inter-tyrosyl radical distances on the order of $33 \AA$ in Ribonucleotide Reductase ${ }^{11}$ and $52 \AA$ in psi factor producing oxygenase $\mathrm{A}^{12}$. In this investigation, we utilized DQC to determine the spatial distribution of tyrosyl radicals in the COX-2 dimer.

Wild type, Y385F, and Y504F human COX-2 constructs were expressed and purified with $0.1 \%$ Tween-20 (v/v) utilized as the solubilization and purification detergent (Supplemental Methods $)^{13}$. The constructs, at concentrations of $150 \mu \mathrm{M}$ monomer, were reconstituted with 
$1.5 \times$ molar excess of heme, followed by the addition of glycerol to a final concentration of $20 \%(\mathrm{v} / \mathrm{v})$. Each construct was then reacted on ice with a 10× molar excess of the alkyl peroxide 15-hydroperoxyeicosatetraenoic acid to generate tyrosyl radicals before flash freezing in liquid nitrogen. DQC data were recorded at $17.3 \mathrm{GHz}$ and $5 \mathrm{~K}$ on a home built Ku-band pulse ESR spectrometer ${ }^{14,15}$ as described in the Supplemental Methods. Distance distributions were reconstructed from the time domain traces using the L-curve Tihkonov regularization method ${ }^{16}$ and refined with the Maximum Entropy method ${ }^{17}$ (see also Supplemental Methods). UV/Vis titration of apo COX-2 with heme was performed to determine the stoichiometry of heme binding (Fig. S3).

In the Y504F construct, tyrosyl radicals can only be generated on Tyr-385, giving rise to a single inter-spin distance across the dimer interface. The distance between Tyr-385 side chains in each monomer in crystal structures of murine (mu) COX-2 is $44.7 \AA$ (Fig $1 \mathrm{~A})^{13}$. The distance distribution determined with DQC for the Y504F construct displays an average distance of $\sim 45 \AA$, corresponding to the distance measured from the crystal structure (Fig 1E). Similarly, radical formation only occurs on Tyr-504 in the Y385F construct. The distance between Tyr-504 side chains across the dimer interface is $58 \AA$ in crystal structures of muCOX-2 (Fig 1B). The distance distribution obtained with DQC for the Y385F construct shows a prominent peak at $\sim 55 \AA$, corresponding closely to the distance measured in the crystal structure (Fig 1F). Taken together, these results confirm that Tyr-385 and Tyr-504 radicals are generated in both monomers of the COX-2 dimer.

Based on the results obtained with the Y385F and Y504F constructs, we predicted that tyrosyl radicals would be generated on Tyr-385 and Tyr-504 in both monomers of the dimer in the wild-type enzyme. This scenario gives rise to several possible inter-spin distances across the dimer interface, as well as an intra-monomer distance between Tyr-385•Tyr504 (Fig 2A). An initial DQC experiment was performed to determine the distribution of tyrosyl radicals across the dimer interface. The resulting distance distribution had a prominent peak at a distance of $\sim 43.4 \AA$, corresponding to the Tyr-385 $\bullet$ Tyr-385 inter-monomer distance and a smaller peak at $\sim 51 \AA$ on its high slope, corresponding to the Tyr-385•Tyr-504 intermonomer distance. No peak that corresponds to the Tyr-504•Tyr-504 inter-monomer distance was resolved, however, the distribution does contain a contribution in this range. A

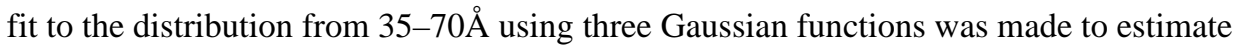
contributions from the three inter-monomer components (Supplementary Methods). The fit depicted in Fig $2 \mathrm{C}$ represents the experiment quite well and accurately reproduces the timedomain DQC signal (Fig. S6). These measurements provide experimental evidence that Tyr-385 and Tyr-504 radicals are generated simultaneously in both monomers of COX-2.

The time domain signal in Fig. 2B is formed by long distances across the dimer interface but also shows the presence of a fast evolving component at the beginning of the record (plotted on expanded scale). Only a short intra-monomer distance can cause this contribution to the signal. Distance reconstruction in Fig. 2C was focused on resolving long distances, with the short distance component being discarded in this distance reconstruction (Fig. S4). Accordingly, an independent DQC experiment was conducted using 2ns dwell time and 200ns data record (Supplemental Methods). The resulting distance distribution is a sharp narrow peak at a distance of $11.2 \AA$, matching the same measured intra-monomer distance 
between Tyr-385 and Tyr-504 in the muCOX-2 crystal structure (Fig. 2E). Resolution of this short distance component further demonstrates that both Tyr-385 and Tyr-504 radicals are generated within a single monomer of COX-2.

Collectively, the DQC experiments provide experimental confirmation that Tyr-385 and Tyr-504 radicals are generated in both monomers of the COX-2 dimer. The distances calculated from the DQC experiments utilizing enzyme in solution are consistent with distances observed when COX-2 is confined to a crystal lattice and sets the stage for future experiments utilizing heterodimers of COX-2 ${ }^{6,13}$. Both DQC and crystallographic characterizations of COX-2 necessitate the use of relatively high concentrations of enzyme and heme $(>50 \mu \mathrm{M})$. Given the low dissociation constant reported for high affinity heme binding to COX-2 ${ }^{6,8}$, the expectation is that both monomers of the sequence homodimer will be chelated with heme. As we observe tyrosyl radical formation in both monomers, heme must be bound to both subunits under the experimental conditions utilized for DQC. Measurements of cyclooxygenase catalysis using an oxygen electrode utilize $5 \mu \mathrm{M}$ heme in the reaction cuvette ${ }^{6,13}$. We further investigated heme binding by titrating COX-2 with heme (Fig. S3). The increase in soret peak absorbance was linear up to $5 \mu \mathrm{M}$ heme indicating saturation of both monomers of COX-2 at low micromolar concentrations. The heme titration result is consistent with both the DQC measurements presented here and previous crystallographic analyses.

To our knowledge this is the first report of distance measurements between pairs of tyrosyl radicals using DQC, as well as an example of accurate measurements of very short distances by pulse ESR. In particular, the Tyr-504•Tyr-504 inter-monomer distance of $\sim 58 \AA$ is at the higher end of distances determined in a membrane protein with pulsed ESR methods, and to our knowledge is the longest distance measured between a pair of protein derived tyrosyl radicals. These experiments highlight the utility of DQC in extracting difficult to measure distance distributions from fast relaxing organic radical cofactors, and the ability of these distance measurements to resolve complex biological questions.

\section{Supplementary Material}

Refer to Web version on PubMed Central for supplementary material.

\section{Acknowledgments}

Funding Sources

This research was supported by grants NIH GM077176 (M.G.M), NIH GM115386 (M.G.M.), NIH P41GM103521 (J.H.F.), and R01EB003150 (J.H.F.).

\section{Abbreviations \\ COX cyclooxygenase \\ NSAID nonsterroidal anti-inflammatory drug \\ AA arachidonic acid}




$\begin{array}{ll}\text { PGG }_{2} & \text { prostaglandin } \mathrm{G}_{2} \\ \text { PGH }_{2} & \text { prostaglandin } \mathrm{H}_{2} \\ \text { ESR } & \text { electron spin resonance } \\ \text { DEER } & \text { double electron-electron resonance } \\ \text { DQC } & \text { double quantum coherence }\end{array}$

\section{REFERENCES}

1. Smith WL, Urade Y, Jakobsson PJ. Enzymes of the cyclooxygenase pathways of prostanoid biosynthesis. Chem Rev. 2011; 111:5821-5865. [PubMed: 21942677]

2. Hata AN, Breyer RM. Pharmacology and signaling of prostaglandin receptors: multiple roles in inflammation and immune modulation. Pharmacol Ther. 2004; 103:147-166. [PubMed: 15369681]

3. Rogge CE, Liu W, Kulmacz RJ, Tsai AL. Peroxide-induced radical formation at TYR385 and TYR504 in human PGHS-1. J Inorg Biochem. 2009; 103:912-922. [PubMed: 19433337]

4. Rogge CE, Liu W, Wu G, Wang LH, Kulmacz RJ, Tsai AL. Identification of Tyr504 as an alternative tyrosyl radical site in human prostaglandin H synthase-2. Biochemistry. 2004; 43:15601568. [PubMed: 14769032]

5. Liu J, Seibold SA, Rieke CJ, Song I, Cukier RI, Smith WL. Prostaglandin endoperoxide H synthases: peroxidase hydroperoxide specificity and cyclooxygenase activation. J Biol Chem. 2007; 282:18233-18244. [PubMed: 17462992]

6. Dong L, Vecchio AJ, Sharma NP, Jurban BJ, Malkowski MG, Smith WL. Human cyclooxygenase-2 is a sequence homodimer that functions as a conformational heterodimer. J Biol Chem. 2011; 286:19035-19046. [PubMed: 21467029]

7. Prusakiewicz JJ, Duggan KC, Rouzer CA, Marnett LJ. Differential sensitivity and mechanism of inhibition of COX-2 oxygenation of arachidonic acid and 2-arachidonoylglycerol by ibuprofen and mefenamic acid. Biochemistry. 2009; 48:7353-7355. [PubMed: 19603831]

8. Dong L, Sharma NP, Jurban BJ, Smith WL. Pre-existent asymmetry in the human cyclooxygenase-2 sequence homodimer. J Biol Chem. 2013; 288:28641-28655. [PubMed: 23955344]

9. Hermanson DJ, Gamble-George JC, Marnett LJ, Patel S. Substrate-selective COX-2 inhibition as a novel strategy for therapeutic endocannabinoid augmentation. Trends Pharmacol Sci. 2014; 35:358367. [PubMed: 24845457]

10. Borbat PP, Freed JH. Measuring distances by pulsed dipolar ESR spectroscopy: spin-labeled histidine kinases. Methods Enzymol. 2007; 423:52-116. [PubMed: 17609127]

11. Bennati M, Robblee JH, Mugnaini V, Stubbe J, Freed JH, Borbat P. EPR distance measurements support a model for long-range radical initiation in E. coli ribonucleotide reductase. J Am Chem Soc. 2005; 127:15014-15015. [PubMed: 16248626]

12. Fielding AJ, Brodhun F, Koch C, Pievo R, Denysenkov V, Feussner I, Bennati M. Multifrequency electron paramagnetic resonance characterization of PpoA, a CYP450 fusion protein that catalyzes fatty acid dioxygenation. J Am Chem Soc. 2011; 133:9052-9062. [PubMed: 21548577]

13. Vecchio AJ, Simmons DM, Malkowski MG. Structural basis of fatty acid substrate binding to cyclooxygenase-2. J Biol Chem. 2010; 285:22152-22163. [PubMed: 20463020]

14. Borbat PP, Crepeau RH, Freed JH. Multifrequency two-dimensional Fourier transform ESR: an X/Ku-band spectrometer. J Magn Reson. 1997; 127:155-167. [PubMed: 9281479]

15. Borbat PP, Georgieva ER, Freed JH. Improved Sensitivity for Long-Distance Measurements in Biomolecules: Five-Pulse Double Electron-Electron Resonance. J Phys Chem Lett. 2013; 4:170_ 175. [PubMed: 23301118]

16. Chiang YW, Borbat PP, Freed JH. The determination of pair distance distributions by pulsed ESR using Tikhonov regularization. J Magn Reson. 2005; 172:279-295. [PubMed: 15649755] 
17. Chiang YW, Borbat PP, Freed JH. Maximum entropy: a complement to Tikhonov regularization for determination of pair distance distributions by pulsed ESR. J Magn Reson. 2005; 177:184-196. [PubMed: 16137901] 

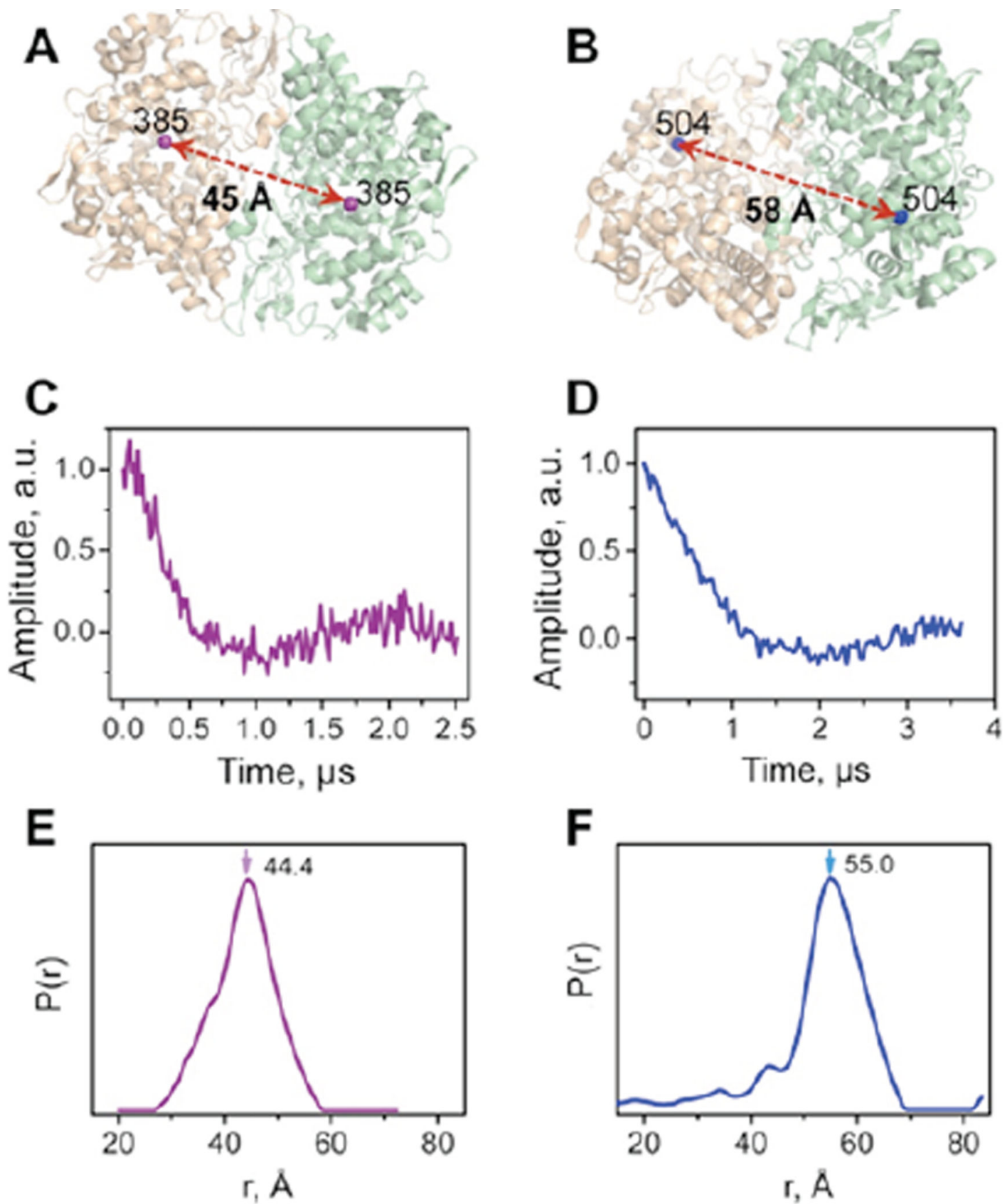

Figure 1. DQC distance measurements between Tyr-385 and Tyr-504 in Y385F and Y504F COX-2

Schematic of the COX-2 dimer, with the locations of (A) Tyr-385 and (B) Tyr-504 depicted as colored spheres. Measured distances between pairs across the dimer interface are indicated and marked with a red dotted line. Time-domain DQC data for (C) Y504F COX-2 and (D) Y385F COX-2. Distance distributions for (E) Y504F COX-2 and (F) Y385F COX-2. 

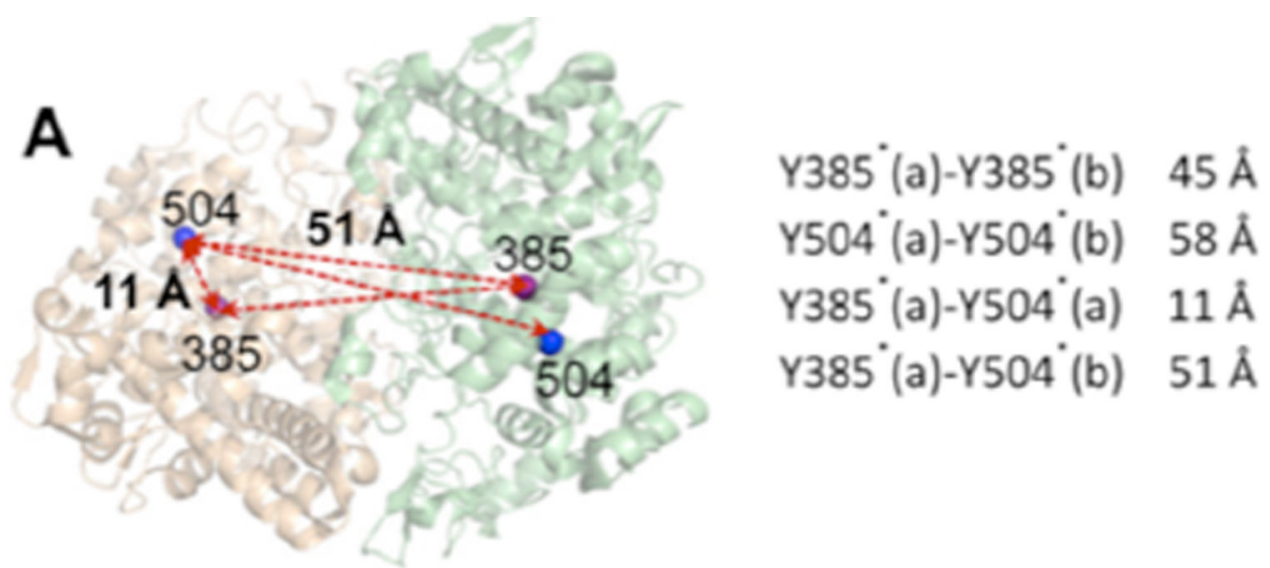

B

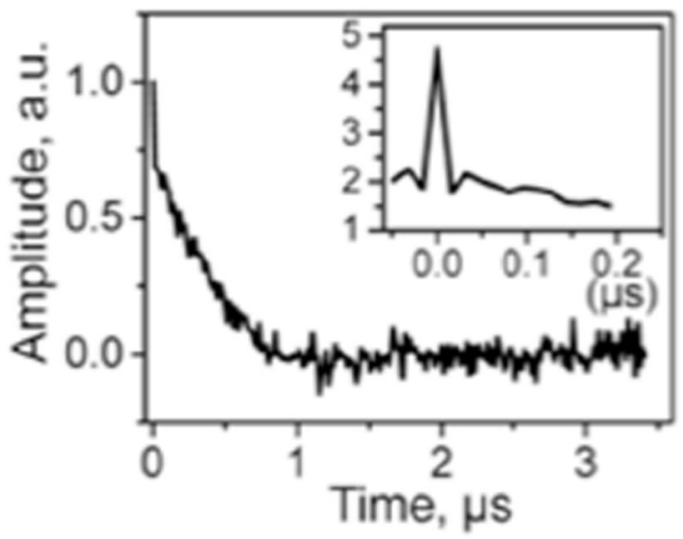

C

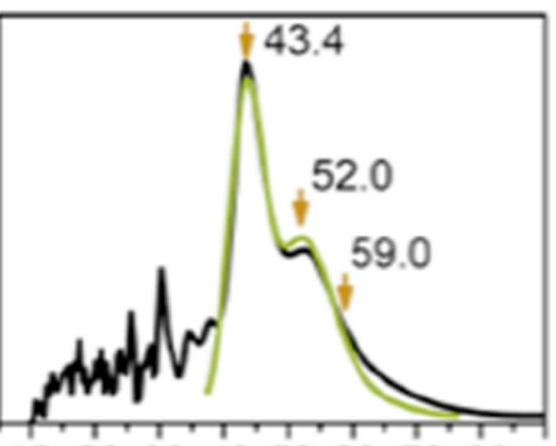

1020304050607080

$$
\text { r, A }
$$

\section{D}
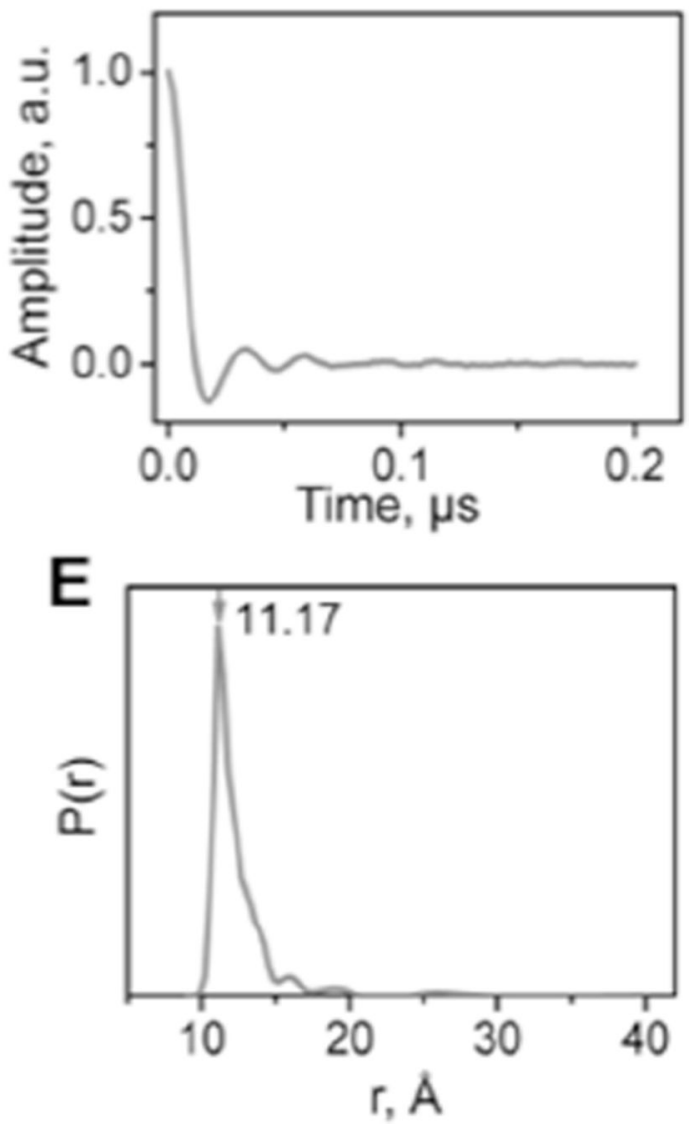

Figure 2. DQC distance measurements between Tyr-385 and Tyr-504 in wild-type COX-2

(A) Schematic of the COX-2 dimer, with Tyr-385 and Tyr-504 depicted as colored spheres.

Estimated distances between pairs across the dimer interface, as well as the single intra monomer distance are indicated. (B) Time-domain DQC data for wild type COX-2 recorded on a $3.5 \mu$ s time scale. Long distances $(>40 \AA)$ from tyrosyl radicals located in opposite monomers dominate the record. The short distance component, originating from Tyr-385 and Tyr-504 located in the same monomer, appears as a single point spike in the beginning of the record and is shown on extended time scale in the insert for clarity. (C) Reconstructed 
distance distributions for the data in plot $\mathbf{B}$. The distance distribution from 35-70 $\mathrm{A}$ was fit to three Gaussian functions with the means indicated by arrows. The short distance component does not appear in the distribution (Supplementary Materials). (D) DQC data for the short distance component in $\mathbf{B}$ were recorded on a short time scale of $0.2 \mu$ s and plotted after removing small linear background (Supplementary Materials). (E) Distance distribution for intra monomer data of panel $\mathbf{D}$ 